\title{
Super-Humeanism and free will
}

\author{
Michael Esfeld \\ University of Lausanne, Department of Philosophy \\ CH-1015 Lausanne, Switzerland \\ Michael-Andreas.Esfeld@unil.ch,www.michaelesfeld.com \\ (forthcoming in Synthese)
}

\begin{abstract}
Super-Humeanism is an even more parsimonious ontology than Lewisian standard Humean metaphysics in that it rejects intrinsic properties (local qualties). There are point objects, but all there is to them are their relative positions (distance relations) and the change of them. Everything else supervenes on the Humean mosaic thus conceived. Hence, dynamical parameters (such as mass, charge, energy, a wave-function, etc.) come in on a par with the laws through their position in the best system. The paper sets out how Super-Humeanism has the conceptual means to reject van Inwagen's consequence argument not by taking the laws to depend on us (as on standard Humean metaphysics), but by taking the initial values of the dynamical parameters that enter into the laws to be dependent on the motions that actually occur in the universe, including the motions of human bodies. The paper spells out the advantages of this proposal.
\end{abstract}

Keywords: consequence argument; free will; Humean metaphysics; David Lewis; SuperHumeanism; Peter van Inwagen

\section{From Lewis's Humean metaphysics to Super-Humeanism}

Consider David Lewis's famous statement of Humean metaphysics, known as Humean supervenience:

It is the doctrine that all there is to the world is a vast mosaic of local matters of particular fact, just one little thing and then another. (...) We have geometry: a system of external relations of spatio-temporal distance between points. Maybe points of spacetime itself, maybe point-sized bits of matter or aether or fields, maybe both. And at those points we have local qualities: perfectly natural intrinsic properties which need nothing bigger than a point at which to be instantiated. For short: we have an arrangement of qualities. And that is all. (Lewis 1986b, pp. IX-X)

There hence are distance relations between point objects (be it space-time points, be it material objects) and natural intrinsic properties instantiated at these points. Candidates for these properties are fundamental physical dynamical parameters such as mass and charge, etc. Everything else supervenes on the configuration of point objects with intrinsic properties of the universe and its change, that is, its evolution. On Lewis's realizer functionalism, supervenience comes down to identity: everything else is realized by - or located in configurations of point objects and their intrinsic properties. ${ }^{1}$

1 See Jackson (1994, p. 25) for this use of the term "location" or "located in". Price (2004) uses the term "placement". 
However, the commitment to local qualities in the guise of intrinsic properties is problematic: the rationale of Lewis's Humeanism is to eschew necessary connections between distinct existences. This implies that the causal role that these properties play is contingent instead of being essential to them. This means that, for instance, the properties of mass and charge can swap their roles: there is another world possible in which the property that we pick out as charge in the actual world plays the role of the property that we pick out as mass in the actual world, and vice versa.

Hence, Lewis's Humeanism implies that properties are pure qualities, known as quiddities. Moreover, we do not have epistemic access to these qualities. Our access is limited to the causal roles that the properties play for the evolution of the configuration of matter in the actual world. ${ }^{2}$ The lack of epistemic access to properties conceived as pure qualities is known as humility. Lewis (2009) endorses both humility and quidditism. However, the rather baroque metaphysics of pure qualities with its implication of possible worlds differing only in the pure qualities that are instantiated in them is a heavy burden on Humeanism, in particular if Humeanism is conceived as a naturalized metaphysics that is supposed to be close to science. ${ }^{3}$

There are two ways out of this situation. The one way is to conceive the properties in such a manner that their essence consists in the causal roles that they play in a world so that they are dispositions or powers; one thereby abandons Humeanism, since there then are primitive modal connections between a disposition or power and its manifestation. ${ }^{4}$ The other way is to reject the commitment to properties altogether and to retain only point objects and distance relations between them. The resulting view has become known as Super-Humeanism, because it goes beyond the already parsimonious ontology of Lewisian Humeanism in also renouncing on local qualities in the guise of intrinsic properties. ${ }^{5}$

Esfeld and Deckert (2017, p. 21) define Super-Humeanism by the following two axioms or principles:

(1) There are distance relations that individuate simple objects - namely matter points.

(2) The matter points are permanent, with the distances between them changing.

By taking the distance relations to individuate the point objects, Super-Humeanism avoids replacing the commitment to pure qualities (quiddities) with a commitment to a primitive stuff essence of matter (bare particulars), or - even worse - primitive thisnisses (haecceities) of the point objects. Moreover, the distance relations are natural relations. More precisely, they are the only natural relations, and they are the world-making relations, since all and only those objects that are related by a distance belong to one and the same world. ${ }^{6}$ Consequently, the Humean mosaic is not arbitrary: on Super-Humeanism, natural relations in the guise of distance relations do what natural properties are supposed to do on Lewis's Humeanism. ${ }^{7}$

See also Jackson (1998, pp. 23-24).

See e.g. Black (2000).

See e.g. Bird (2007).

5 See Esfeld and Deckert (2017, ch. 2). See Hall $(2009, \S 5.2)$ as well as Loewer (2007) for forerunners of Super-Humeanism. See Wilson (2018), Marmodoro (2018), Darby (2018), Lazarovici (2018), Matarese (2019) and Simpson (2019) for critical discussions of Super-Humeanism.

6 See also Lewis (1986a, pp. 76-78).

7 Such an arbitrariness threatens, by contrast, the "package deal account" proposed by Loewer (2007) that is also directed against Lewis's natural properties. 
Again, everything else then supervenes on the Super-Humean mosaic of the configuration of point objects of the universe and the change in the distances between the point objects throughout the history of the universe.

Super-Humeanism arguably is superior to Lewis's Humeanism in that it avoids the commitments to quidditism and humility. Furthermore, Esfeld and Deckert (2017) show in detail how Super-Humeanism accommodates modern physics and is, in particular, not hit by the stock objections to Humean supervenience from quantum physics. The argument of this paper is that Super-Humeanism also is superior to standard, Lewisian Humean metaphysics in the way in which this stance can include free will. To make this case, I first consider van Inwagen's famous consequence argument and the Humean reply to it (section 2). I then set out how Super-Humeanism deals with that argument (section 3) and sum up the arguments for Super-Humeanism in that respect in a brief conclusion (section 4).

\section{Van Inwagen's consequence argument and the Humean reply}

Consider the following informal statement by Peter van Inwagen of his famous consequence argument:

If determinism is true, then our acts are the consequences of the laws of nature and events in the remote past. But it is not up to us what went on before we were born, and neither is it up to us what the laws of nature are. Therefore the consequences of these things (including our present acts) are not up to us. (van Inwagen 1983, p. 16) ${ }^{8}$

This argument can be construed as follows:

(1) If determinism is true, then our acts are the consequences of the laws of nature and events in the remote past.

(2) It is not up to us what went on before we were born.

(3) It is not up to us what the laws of nature are.

(4) From (1)-(3): The consequences of these things (including our present acts) are not up to us.

(5) If our present acts are not up to us, we do not have free will.

(6) Determinism implies that we do not have free will.

The argument is not about determinism in particular. It could, for instance, not be avoided by endorsing a version of quantum physics with indeterministic wave-function collapse. The argument goes through as long as there are universal laws of nature, be they deterministic or not. If the laws are probabilistic, then the objective probabilities for our acts are the consequences of the laws of nature and events in the remote past. It then follows that if what fixes these probabilities does not include anything that is up to us, we do not have free will. ${ }^{9}$

Furthermore, what is at issue here is not a view of laws according to which the laws somehow produce or bring about the evolution of the configuration of matter of the universe. The term "consequences" in the consequence argument can simply be read as logical consequences: on determinism, given the propositions stating the laws of nature and the propositions describing the state of the world at an arbitrary time in the sense of the propositions describing the initial conditions that enter into the laws, the conjunction of these propositions entails the propositions describing the state of the world at any other time (if the laws are probabilistic, we get to propositions stating objective probabilities for what there is 
in the world at other times). The entailed propositions include those ones that describe the state of motion of the particle configurations that are human bodies. No one is in the position to deduce these propositions and thus to make deterministic predictions about the motions of human bodies. But this is irrelevant to the argument: it is about ontology and not about the possibility of predictions. Furthermore, also the propositions about past states of motion are thus entailed. But, again, this does not touch upon the truth of premise (1). Its truth does not depend on any particular stance in the metaphysics of laws of nature. It hence hits also Humeanism about laws.

One possible reply to this argument that is available to Humeanism (but also to other stances in the metaphysics of nature) is to dismiss premise (4), that is, to deny that the laws of nature and the initial conditions of the universe on which we have no influence imply that our present acts are not up to us. In this case, one defends the most widespread form of a compatibilism of determinism and free will: one endorses (1) to (3) and modifies (4) and (5) such that a case is made for the implications of the laws and initial conditions being compatible with us having free will. ${ }^{10}$

The idea behind premises (4) and (5) is that if persons have free will, in those situations in which a person exercises her free will, her acts are up to her. This means that she could have done otherwise. Hence, if in a given situation a person could not have done otherwise, her acts have not been up to her and she did not have free will in that situation. The credibility of such a compatibilism of (deterministic) laws of nature and free will then depends on putting forward a plausible restriction of the clause "could have done otherwise" in terms of "could have done otherwise if the circumstances that led to her action had been otherwise". The problem with the plausibility of any such restrictions is that, at the end of the day, they come down to saying that the person could have done otherwise only if the laws of nature or the initial conditions of the universe that enter into the laws had been different. But this, then, just is what drives the reservations against this attempt to make free will compatible with determinism: however sophisticated an elaboration of restrictions of the clause "could have done otherwise" one may develop, it is true on any version of such a compatibilism of free will and determinism that our acts are the consequences of the laws of nature and the initial conditions of the universe, on which we have no influence according to (2) and (3).

To put it differently, a position that seeks to qualify the clause "could have done otherwise" is well taken in pointing out that free will has to be distinguished from pure chance events in nature, that is, the idea of events that occur such that there are not even objective probabilities for their occurrence, because they do not come under any salient regularity. Thus, stressing that our actions are up to us or that, in exercising free will, persons could have done otherwise is not sufficient as a theory of free will. Any such theory has to spell out what distinguishes an action being up to us or a person's ability to do otherwise from pure chance events. Developing such a conception of free will is beyond the scope of this paper. This paper is only about exploring the conceptual resources that are particular to Super-Humeanism in rebutting the consequence argument. In sum, if the qualification of the clause "could have done otherwise" cannot remove the implication that our acts are the consequences of the laws of nature and the initial conditions of the universe on which we have no influence according 
to (2) and (3), the intuition is well-founded that there is no conceptual space left to establish a significant free will.

Hence, if the backbone of science, namely universal laws of nature, is not to contradict human free will, we have to call into question premise (2) and / or premise (3) of the consequence argument. That is, we need a conception of laws of nature from which it follows that the laws and / or the initial conditions of the universe are in a certain sense "up to us". Furthermore, such a conception of laws has to be backed by cogent arguments that are independent of the issue of human free will. Moreover, it should be situated within scientific realism: science discovers laws and initial conditions. They are not social constructions or in any way dependent on observers.

On Humeanism about laws of nature, premise (3) is false. The reason is that first comes the motion of the matter in the universe, then come the laws as the theorems of the system that strikes the best balance between being simple and being informative in representing the motion that actually occurs (i.e. the best system). Our bodily motions are part of the motion of the matter in the universe, albeit only a very tiny part. Nonetheless, they are thereby part of the basis that fixes the laws of nature. Thus, Jenann Ismael writes:

When we adopt a globalist perspective, our activities become part of the pattern of events that make up history. Since our activities partly determine the pattern, and the pattern determines the laws, our activities partly determine the laws. (Ismael 2016, p. 111; see also pp. 225-226)

In that sense, the laws are "up to us". Hence, if persons had chosen to do otherwise, the laws of nature would have been slightly different. ${ }^{11}$

Note that this compatibilism of free will and (deterministic) laws of nature within standard Humean metaphysics follows from Humeanism about laws only - that is, it follows from the claim that the laws supervene on the mosaic alone, with our bodily motions being part and parcel of the mosaic. Consequently, this compatibilism is not tied to a particular theory of counterfactuals. It entails counterfactuals of the type that if persons had chosen to do otherwise, the laws of nature would have been slightly different; but it is not committed to a specific theory of counterfactuals. Hence, it is different from Lewis's own reply to van Inwagen's consequence argument (Lewis 1981), which, in turn, does not invoke Humeanism about laws, but rests on Lewis's theory of counterfactuals such that, in brief, a person having done otherwise would constitute a law-breaking event in the guise of a local miracle.

There are strong arguments in favour of Humeanism about laws of nature that are independent of the issue of free will. ${ }^{12}$ The upshot then is that this stance gives us all that motivates endorsing laws of nature in a scientific realist spirit - such as the significance of laws for explanations, for supporting counterfactuals, etc. - without going beyond a parsimonious ontology of matter in motion, that is, without calling for a commitment to primitive modal entities. However, the drawback in the context of free will is that this conception seems to give us too much: there is a well-founded sense in which the laws of nature define what we can do and what we cannot do. More precisely, we need laws of nature to delimit the range within which we can freely choose our actions. For instance, a person can choose to walk slowly or quickly, but she cannot choose to travel faster than light. By the

See also Beebee and Mele (2002) for a detailed argument. See already Swartz (2003, ch. 11, in particular p. 127). 
same token, she can choose to walk left or right, but she cannot choose to jump as high as her house without technical aid.

This difference can be taken into account within Humeanism about laws: there are stable patterns or regularities of motion in the universe. Only if there are such stable patterns or regularities are there laws at all. Keeping these patterns or regularities as they are expressed in the laws of nature that we formulate fixed, it is physically or nomologically impossible for a person to travel faster than light, or to jump as high as her house without technical aid. This is all that is needed for the laws of nature to define the range within which we can act freely. That notwithstanding, it is metaphysically possible that, tomorrow, a person travels faster than light or jumps as high as her house without technical aid. There is nothing in the present or past configuration of matter that makes it impossible for such things to happen. It is only that if we presume that the salient regularities that were discovered in the past motion of matter will prevail in the future that we get to such restrictions. But there is nothing in the universe that makes it necessary that these regularities will prevail in the future. ${ }^{13}$ Deliberating about her actions on the basis of observed regularities of motion as expressed in the laws of nature that figure in our scientific theories is in any case all that a person can do, independently of whether or not there is something in nature that constrains the future evolution of the configuration of matter.

In this way, one can seek to draw a distinction between metaphysical possibility and nomological or physical impossibility within Humeanism about laws. Nonetheless, however convincing or not this reply may be, ${ }^{14}$ the following impression remains well taken: if one has to attack premise (2) or premise (3) of van Inwagen's consequence argument in order to show why universal laws of nature do not enter into conflict with human free will, it is in the first place premise (2) about the initial conditions not being up to us that comes under scrutiny. One calls into question premise (3) according to which the laws of nature are not up to us only as a last resort, precisely because the laws of nature delimit the range of what we can do, whatever the merits of Humeanism as a metaphysics of laws of nature may otherwise be.

\section{Super-Humeanism, dynamical parameters and free will}

On Super-Humeanism, premise (2) of the consequence argument is false. The reason is that Super-Humeanism treats the dynamical parameters on a par with the laws as supervening on the mosaic of point particles individuated by their relative distances and the change of these distances. The ontological primitives are thus only the point particles as individuated by the distance relations in which they stand and the change in these relations. Hence, neither a geometry with a particular metric (such as Euclidean geometry) nor dynamical parameters such as mass, charge, a wave-function, etc. count among the ontological primitives. As regards the latter, already Ernst Mach emphasized when commenting on Newton's Principles, "The true definition of mass can be deduced only from the dynamical relations of bodies" (1919, p. 241). Hence, mass is a parameter that expresses a dynamical relation among the physical objects. It is introduced in a functional manner in terms of the causal role that it exercizes for the motion of matter. It can therefore be taken to be realized by - or located in the motion of matter as defined by the change in the distance relations among featureless point objects only. The same goes for charge, energy, a wave-function, etc.

13 See again Beebee and Mele (2002, pp. 209-217).

14 See Hüttemann and Loew (2019) for an argument that it is not convincing. 
That is to say: particles are not intrinsically electrons, protons, neutrons, etc. Some particles are electrons because they move electronwise so to speak, etc. They are not intrinsically divided into various particle kinds. Only their contingent, overall motion makes it that they come under specific particle kinds. Obviously, this is ontology and not the view that working physicists take when seeking for a simple representation of the motion of matter; regarding the particles as being ab initio divided into several species greatly simplifies the representation of their evolution. However, when it comes to ontology, economic representation is not a good guideline, since one is then committed to attributing intrinsic properties to the particles that are either pure qualities - and thus quiddities - or dispositions and hence come with a primitive modality.

By the same token, the geometry in the sense of a specific metric (such as e.g. Euclidean geometry) can be taken to come in through the role that it plays for the motion of matter. Thus, Huggett (2006) shows how geometry and inertial frames in classical mechanics can be received as figuring in the best system that describes the change in the spatial relations among point particles instead of being ontological primitives that require a commitment to absolute space or space-time and absolute magnitudes.

Hence, on Super-Humeanism, the geometry of space-time as well as the dynamical parameters such as mass, charge, spin, a wave-function, etc. come in through their role in the representation of the evolution of the ontological primitives - that is, the evolution of the distance relations in the particle configuration of the universe. They all are the means to achieve a representation of that evolution in terms of laws of nature that are both simple and informative. In a nutshell, they all come in as a package together with the laws. ${ }^{15}$

Consequently, whether or not a given particle or particle configuration realizes the role that functionally defines a certain dynamical parameter is a holistic affair: it depends on how the particle or the particle configuration in question moves within the whole particle configuration of the universe. Thus, the propositions that ascribe certain values of, for instance, mass and charge to individual particles are not true in virtue of these being intrinsic features of the particles. They are true in virtue of certain stable regularities in the overall particle motion. That is to say: the state of the universe at any given time, which enters as initial condition into the laws, contains elements that are not intrinsic to what there is at that time, but depend in the last resort on the overall change in the universe. In other words, they depend on the entire temporal evolution of the configuration of matter of the universe. These are notably the initial values of parameters such as mass, charge, the wave-function of the universe, constants of nature, etc. In order for these parameters to play their role to simplify the account of the motion that occurs in the universe, what role these parameters play and, notably, what has to be put in as their initial values, depends on the change that actually happens in the universe. What these values are is fixed by "the dynamical relations of bodies", as already Mach (1919, p. 241) put it with respect to mass in Newtonian mechanics.

To stress again, on Super-Humeanism, the correct value of these parameters that enters into the state of the universe at any given time depends not only on what motion happens in the universe earlier than that time, but also on what happens later than that time. The reason is that these parameters are not located in the particle motion at any given time. They are located only in the overall particle motion throughout the history of the universe. Thus, to put it in a 
nutshell, we are ignorant of, for instance, the initial wave-function of the universe at the initial state of the universe not only because of a limit on what is accessible to us, but also because what is the initial wave-function of the universe is only fixed at the end of the universe so to speak.

The ontological primitives of distance relations individuating point particles and change in these relations are not sufficient to define an initial state of the universe that enters into a law of nature. To put it differently, no law of nature can be formulated by using only the primitive predicates of "point particles", "distance relations" and "change of distance relations". The reason is that no configuration of point particles as defined only by their relative distances contains information about how the configuration changes - more precisely, how it can and cannot change -, given the fact of change occurring.

To be able to extract information about the evolution of the distance relations among the point particles, further parameters are required that contain such information because they are defined in terms of a functional role that they play for the change of the distance relations in the configuration of matter of the universe - such as gravitational mass being defined in terms of a role for attractive motion, etc. These parameters, then, yield an initial state such that laws can operate by being fed with such initial states. These laws may then be deterministic in the sense that given propositions that describe an arbitrary such initial state plus the propositions that express the laws, the propositions that describe the state of the universe at any other time are entailed. However, there is no question of determinism based only on the primitive predicates of point particles individuated by relative distances and change of distances. For laws - and a fortiori deterministic laws - to operate, predicates that are defined in terms of a functional role for the evolution of the configuration of matter are indispensable. According to Super-Humeanism, such predicates do not designate or refer to anything that is intrinsic to any given configuration of matter. Their determinate values - more precisely the determinate values of the physical parameters in question - obtain in virtue of the actual, overall evolution of the configuration of matter as defined by the above-mentioned primitive predicates.

Consequently, on Super-Humeanism, the bodily motions of human beings that are expressions of their free will contribute to determining the values of the dynamical parameters at the initial state of the universe, that is, the state that enters as initial condition into the laws and without which the laws would not be in the position to describe the evolution of the universe. Hence, if human beings chose to do otherwise, in the first place, slightly different initial values for the dynamical parameters at the initial state of the universe would have to be figured out in order to achieve a system that maximizes both simplicity and informational content about the change that actually occurs in the universe.

For the sake of illustration, assume that quantum mechanics is the correct theory of the universe. Then, what would be slightly different if humans chose to do otherwise than they actually did would not be the Schrödinger equation (and / or the guiding equation of Bohmian quantum mechanics or a law of wave-function collapse - depending on which version of quantum mechanics one endorses in order to solve the measurement problem), but the initial quantum state of the universe. That is, the values that the universal wave-function takes as initial condition in the configuration space of the universe would be slightly different in that case. Note that the quantum state of the universe is not the state as given by the ontological primitives that make up the Humean mosaic (such as relative positions of point particles), but the state as given by an initial wave-function. However, what the wave-function is at a time 
can be taken to depend on the entire evolution of the configuration of matter. Like mass and charge, one can regard the wave-function as a dynamical parameter that is defined by its functional role for the evolution of matter and thereby realized by or located in that evolution as a whole. This stance is known as quantum Humeanism. ${ }^{16}$

In that way, van Inwagen's consequence argument turns out to be invalid without the Super-Humean being committed to saying that it is up to us what the laws of nature are. Instead, there is an ambiguity in the phrase "it is not up to us what went on before we were born": this statement may mean that we cannot change what happened in the universe before our birth. In that sense, the Super-Humean endorses this statement. But this statement can also include reference to an initial state of the universe before we were born. Insofar as that initial state enters into laws of nature, it includes values of parameters that are not intrinsic to it. These values depend on what happens later in the universe, including the particle motions that are expressions of human free will. Van Inwagen has to use this statement in that latter sense. Otherwise, we would not get to steps (4), (5) and (6) of the argument. The past particle motions have consequences for the future, including our present acts, only if they include values of additional dynamical parameters over and above position that enter as initial conditions into the laws.

Consequently, rejecting premise (2) does not imply that human decisions about how to move their bodies alter past particle positions and motions. This distinguishes the present proposal from proposals in terms of backwards causation. ${ }^{17}$ The particle positions and motions before we were born are what they are, independently of what we do. The crucial point to note in that respect is that all observations, including all measurement records, are position observations and are recorded as spatial configurations. Human free will does not alter past observations or touch upon the validity of records of the past. However, there is a distinction between position as primitive parameter and the additional dynamical parameters that enter into the initial conditions for a law of motion. These latter are functionally defined in terms of the role that they play for the motion that actually occurs. Their initial values can therefore be dependent on future motions, including motions that are the result of free will, without any paradox arising. They are realized by or located in the motion as a whole.

Again, this proposal does not depend on a particular theory of counterfactuals. This proposal how to make free will compatible with laws of nature, which can be deterministic, only exploits the Super-Humean idea according to which the dynamical parameters that define a state that enters as initial condition into a law depend on the motions that actually happen in the universe, including the motions of human bodies. Of course, this proposal then implies counterfactuals to the effect that if persons had chosen to do otherwise, the initial values of some of these parameters at the initial state of the universe would have been slightly different; but it remains neutral on the theory of counterfactuals.

The present proposal is also distinct from the way in which premise (2) is rejected in proposals that are situated within the metaphysics of a block universe and that are combined with Humeanism about laws, so that premise (3) comes out false as well. ${ }^{18}$ In these proposals, premise (2) is dismissed only because (i) all events exist in a timeless manner and (ii)

16 See Miller (2014), Esfeld (2014), Callender (2015) and Bhogal and Perry (2017).

17 See Forrest (1985) for one such proposal.

18 For such proposals, see Hoefer (2002) and Ismael (2016, ch. 6 and pp. 227-230). See Brennan (2007) for a criticism of Hoefer's proposal. 
determinism implies that, given the complete description of the state of the universe at an arbitrary time (which may be a time at which persons live and act) plus the laws, also the propositions about all the past states of the universe are entailed. But this is not sufficient to dismiss the consequence argument. It does not touch upon the concern that given the complete description of what there was when the initial state of the universe occurred plus the propositions stating the laws of nature, these propositions entail the propositions about everything that humans do later in the evolution of the universe.

The present proposal does not tie Humeanism to the metaphysics of a block universe. It acknowledges that the issue of free will is not one of static dependency or determination relations among timeless events, but whether free will makes it that certain rather than other changes happen (such as me having coffee instead of tea for breakfast). Its point is that this can be fully granted in a Humean metaphysics, while still taking the laws of nature to define the range within which we can choose what to do. The present proposal admits change as a primitive. It rejects premise (2) of the consequence argument in a differentiated manner: the values of only some of the parameters that enter into the initial conditions of the universe according to a physical theory depend on the actual change that occurs in the universe, namely those that are defined in terms of their functional role for the evolution of the primitive parameters. But this does not hold for the values of the primitive parameters (i.e. relative positions and their change endorsed as primitive). We thereby obtain a sense in which a part of the initial conditions of the universe that enter into laws of nature is "up to us" in a manner that is pertinent to free will: the values of the parameters that are defined in terms of their functional role for the evolution of the primitive parameters did not yet exist when the initial state of the universe occurred long before we were born. They are not intrinsic to that state, but are realized by or located in the entire motion of the matter of the universe.

A similar consideration applies to the laws in the special sciences, such as laws in genetics, evolutionary biology, neuroscience, etc. Again, even leaving aside the fact that if these laws were deterministic, they would always apply only against the background of normal conditions, an alleged genetical, evolutionary biological or neurobiological determinism would only hold on the basis of specifying appropriate initial conditions. However, parameters enter into these initial conditions that are defined by their biological or neurobiological function. Consequently, one can apply the outlined procedure also to the dynamical parameters that figure in the laws of the special sciences and that are defined in terms of their functional role for the evolution of the systems to which they apply. These parameters then are realized by or located in the evolution of these systems. Their initial values thereby depend on the actual evolution of these systems. Hence, it follows that some of the values of parameters that enter into these initial conditions depend on what humans choose to do out of their free will. Consequently, if humans had chosen to do other things than they actually did, some of the initial values of these parameters applying to states that occurred before the human actions in question happened would have been slightly different.

Furthermore, on Humeanism about laws, special sciences' laws are again just a pattern in the phenomena, instead of something that governs, produces or brings about the phenomena. Thus, as a matter of fact, human decisions and bodily motions may manifest certain stable regularities with respect to genes, neuronal configurations, etc. Nevertheless, the decisions and bodily motions come first and then come the regularities that they may manifest or not. 
Premise (3) of the consequence argument - the laws of nature are not up to us - comes out false in a scientific realist framework if and only if one adopts Humeanism about laws. For premise (2) - the initial conditions are not up to us - to be proven wrong, one has to make the step from Humeanism to Super-Humeanism. One has to reject notably the idea that the dynamical parameters refer to intrinsic properties of the objects to which they are attributed. However, premise (2) can turn out to be false also if one eschews a commitment to Humeanism about laws. It is sufficient for premise (2) to be dismissed that one endorses what Super-Humeanism says about the dynamical parameters. For that, one only has to subscribe to the definition of these parameters in terms of their functional role for the evolution of the ontological primitives. More precisely, one has to regard these definitions as being such that they locate these parameters in the ontological primitives, such as the particle motion that actually occurs. If these parameters are thus located (or realized), they are nothing over and above the particle motion. Consequently, their determinate values are not yet there at the initial particle positions and their change. They come out of the particle motion.

This stance can therefore also go with an anti-Humean view of laws of nature according to which the laws are ontological primitives that pose a general constraint on how the matter in the universe can move without actually producing the motion of matter. One may prefer such a view of the laws in order to anchor the scope that the laws of nature define for our actions in the ontology. What a person can and what she cannot do is any case fixed by the laws of nature, be they ontological primitives or be they reducible to the salient regularities of the motion of matter that hold throughout the universe. A person can go left or right, but she cannot jump as high as her house without technical aid. The point of rejecting premise (2) rather than premise (3) of the consequence argument, whatever stance one takes in the metaphysics of laws, is this one: taking determinism for granted, where the person goes does not depend on the laws as such, but on the initial values of the dynamical parameters over and above those defining a primitive parameter (such as particle positions and their change) that enter as initial condition into the laws; and these values, in turn, are not fixed before the person decides to go left or right, but depend on her decision among other things.

\section{Conclusion}

To conclude, this paper is an argument for the following three claims:

(1) The recently developed stance of Super-Humeanism entails an original rebuttal of van Inwagen's consequence argument and thereby a new version of a compatibilism of deterministic laws of nature and free will, which is based on a differentiated rejection of the premise according to which the initial conditions of the universe are not up to us. Whatever its merits or demerits may be, this proposal deserves to be recognized as a stance of its own on the issue of how free will can be compatible with universal laws of nature.

(2) By dismissing the premise according to which the initial conditions of the universe are not up to us, the present proposal does not come into conflict with the well-founded widespread view according to which the laws delimit the range within which we can freely choose our actions. More precisely, what a person does in a given concrete situation (or what are the objective probabilities for what she does) is not determined by the laws as such, but by the values of the dynamical parameters that enter into the initial conditions. Super-Humeanism addresses precisely this point in taking these values to be dependent on 
the overall evolution of the configuration of matter of the universe, which includes the human bodily motions. This is an important advantage in comparison to the compatibilism within standard Humean metaphysics (Humeanism about laws), which rests only upon the premise of the laws being dependent also on the human bodily motions and does not address the issue of the initial values.

(3) The rationale of Super-Humeanism is ontological parsimony: there is only one type of natural relations that are world-making relations, namely distance relations individuating point particles, and change in these relations. There are no intrinsic properties. The distinctively Super-Humean compatibilism of determinism and free will follows from this ontological parsimony alone. It thereby has the advantage of not being tied to Humeanism about laws, that is, of being also available in case one has reservations about the Humean rejection of any sort of constraint that the laws put on the evolution of the configuration of matter of the universe.

In sum, the stock argument for an incompatibility between free will and deterministic laws in science (more generally speaking, universal laws) fails, because the laws require initial conditions to operate. Into these initial conditions enter parameters whose initial values depend, according to Super-Humeanism, on the motion of matter that actually occurs in the evolution of the universe, including the motions that are the expressions of human free will.

Acknowledgements: I would like to thank the organizers and the participants of the workshop "Humeanisms" in Budapest in July 2018 as well as three anonymous referees for their helpful comments on the draft of this paper.

\section{References}

Beebee, Helen and Mele, Alfred R. (2002): "Humean compatibilism”. Mind 111, pp. 201-223.

Bird, Alexander (2007): Nature's metaphysics. Laws and properties. Oxford: Oxford University Press.

Black, Robert (2000): “Against quidditism”. Australasian Journal of Philosophy 78, pp. 87-104.

Bhogal, Harjit and Zee, Perry (2017): “What the Humean should say about entanglement”. Noûs 51, pp. 74-94.

Brennan, Jason (2007): "Free will in the block universe". Philosophia 35, pp. 207-217.

Callender, Craig (2015): “One world, one beable”. Synthese 192, pp. 3153-3177.

Darby, George (2018): “A minimalist Humeanism?”. Metasience 27, pp. 433-437.

Esfeld, Michael (2014): “Quantum Humeanism”. Philosophical Quarterly 64, pp. 453-470.

Esfeld, Michael and Deckert, Dirk-André (2017): A minimalist ontology of the natural world. New York: Routledge.

Forrest, Peter (1985): "Backward causation in defence of free will”. Mind 94, pp. 210-217.

Frankfurt, Harry G. (1971): "Freedom of the will and the concept of a person". Journal of Philosophy 68, pp. 520.

Hall, Ned (2009): "Humean reductionism about laws of nature". Unpublished manuscript, http://philpapers.org/rec/HALHRA

Hoefer, Carl (2002): "Freedom from the inside out". Royal Institute of Philosophy Supplement 50, pp. 201-222.

Hüttemann, Andreas and Loew, Christian (2019): "Freier Wille und Naturgesetze - Überlegungen zum Konsequenzargument”. In: K. von Stoch, S. Wendel, M. Breul and A. Langenfeld (eds.): Streit um die Freiheit - philosophische und theologische Perspektiven. Paderborn: Mentis. Pp. 77-93.

Huggett, Nick (2006): “The regularity account of relational spacetime”. Mind 115, pp. 41-73.

Ismael, Jenann (2016): How physics makes us free. Oxford: Oxford University Press.

Jackson, Frank (1994): “Armchair metaphysics”. In: J. O’Leary-Hawthorne and M. Michael (eds.): Philosophy in mind. Dordrecht: Kluwer. Pp. 23-42. 
Jackson, Frank (1998): From metaphysics to ethics. A defence of conceptual analysis. Oxford: Oxford University Press.

Lazarovici, Dustin (2018): "Super-Humeanism: a starving ontology". Studies in History and Philosophy of Modern Physics 64, pp. 79-86.

Lewis, David (1981): “Are we free to break the laws?”. Theoria 47, pp. 113-121. Reprinted in Lewis (1986b), pp. 291-298.

Lewis, David (1986a): On the plurality of worlds. Oxford: Blackwell.

Lewis, David (1986b): Philosophical papers. Volume 2. Oxford: Oxford University Press.

Lewis, David (2009): “Ramseyan humility”. In: D. Braddon-Mitchell and R. Nola (eds.): Conceptual analysis and philosophical naturalism. Cambridge (Massachusetts): MIT Press. Pp. 203-222.

Loewer, Barry (1996): "Freedom from physics: quantum mechanics and free will”. Philosophical Topics 24, pp. 91-112.

Loewer, Barry (2007): “Laws and natural properties”. Philosophical Topics 35, pp. 313-328.

Mach, Ernst (1919): The science of mechanics: a critical and historical account of its development. Fourth edition. Translation by Thomas J. McCormack. Chicago: Open Court.

Marmodoro, Anna (2018): “Atomism, holism and structuralism: costs and benefits of a minimalist ontology of the world”. Metasience 27, pp.421-425.

Matarese, Vera (2019): “A challenge for Super-Humeanism: the problem of immanent comparisons". Forthcoming in Synthese, DOI 10.1007/s11229-018-01914-y

Miller, Elizabeth (2014): "Quantum entanglement, Bohmian mechanics, and Humean supervenience". Australasian Journal of Philosophy 92, pp. 567-583.

Price, Huw (2004): "Naturalism without representationalism." In: M. de Caro and D. Macarthur (eds.): Naturalism in question. Cambridge (Massachusetts): Harvard University Press. Pp. 71-88.

Simpson, William M. R. (2019): "What's the matter with Super-Humeanism?”. Forthcoming in British Journal for the Philosophy of Science, DOI 10.1093/bjps/axz028

Swartz, Norman (2003): The concept of physical law. https://www.sfu.ca/ swartz/physical-law/index.htm First edition Cambridge: Cambridge University Press 1985.

van Inwagen, Peter (1975): “The incomptability of free will and determinism”. Philosophical Studies 27, pp. 185-199.

van Inwagen, Peter (1983): An essay on free will. Oxford: Oxford University Press.

Wilson, Alastair (2018): "Super-Humeanism: insufficiently naturalistic and insufficiently explanatory". Metasience 27, pp. 427-431. 